\title{
SULP (SUSTAINABLE URBAN LOGISTICS PLAN) AS A TOOL FOR SHAPING SUSTAINABLE URBAN LOGISTICS: A REVIEW OF EUROPEAN PROJECTS SUPPORTING THE CREATION OF SULP
}

\begin{abstract}
Few European cities have logistics plans. However, many European projects involve cities to create such plans and share the results with others. This article reviews the most important projects regarding the creation of Sustainable Urban Logistics Plans (SULP) as the fundamental element of all logistics plans. Having a sustainable logistics plan is necessary to achieve the ambitious goals set in the White Paper on Transport - zero-emission logistics in cities by 2050.

Keywords: SULP, SUMP, sustainable urban logistics plan, sustainable urban mobility plan, novelog, sulpiter, enclose

JEL: R5

\section{Introduction}

Transport is the second largest energy-intensive sector, with $32 \%$ share in final energy consumption. In the 2001 White Paper on Transport, the European Commission presented an ambitious goal of CO2-free urban logistics to 2030. Eighty-two percent of Europeans will live in cities by 2050, so it is important to address city transport issues in functional urban areas (FUAs), taking into account the functional transport and economic relations between urban centres and surrounding metropolitan areas. This way, the developed strategies will have an impact on the territorial and economic development of urban regions in Europe. Sustainable Urban Logistics Plan (SULP) is a policy-support tool aimed at a large number of cities in Europe that may not have the resources to undertake a serious policy evaluation
\end{abstract}


and modelling of work for sustainable urban logistics. The SULP methodology is connected to the Sustainable Urban Mobility Plan (SUMP), which has been widely implemented in many European cities. The article summarizes the results of NOVELOG, ENCLOSE, and SULPiTER projects.

\section{Methodology and theory}

When analysing the preparation of European cities for urban logistics planning, the desk research method was used, which involved analysing, verifying, and merging existing data and information from the results of European projects.

The collected material was ordered and presented in the form of a summary. The paper can be used as a road map to proceed with planning urban logistics in cities and to create Sustainable Urban Logistics Plans.

\section{Results}

The most important conclusions include:

- Few cities in Europe have a logistics plan,

- There is no set of measures that suits every city,

- The action plan, however, can serve all kinds of cities.

\section{Discussion}

The main elements of SULP understand the participatory approach and political engagement. A bottom-up approach is assumed, starting with the needs of users, the requirements of operators, and urban objectives. This methodology has been used (and tested) by the nine cities of the ENCLOSE Project and seven cities of SULPiTER project to develop local SULPs and is now available for eventual adoption by other European cities willing to address the issues of urban transport in general urban mobility planning.

ENCLOSE (ENergy efficiency in City LOgistics Services) for small and mid-sized European Historic Towns is a project funded by the European Commission under the Intelligent Energy - Europe (Intelligent Energy Europe - IEE) programme, which supports efforts to increase energy efficiency. The ENCLOSE project started in May 2012, and its implementation lasted until November 2014. The main objective of the project was to raise awareness about the challenges faced by energy efficient and sustainable urban logistics in small and medium-sized historical cities in Europe and about concrete opportunities to achieve significant improvements and benefits through the implementation and application of appropriate and effective measures specifically adapted to such urban environments. Certain solutions and innovative programmes already exist in Europe, proving that this approach is feasible and beneficial. Using and developing existing experiences, the ENCLOSE project 
will help to eliminate potential barriers, promote possible solutions for many small and medium-sized historical European cities, and explore and demonstrate the possibility of transferring these solutions among cities. The project was planning to promote and make future use of energy-efficient and sustainable solutions for urban logistics in as many small and medium-sized historical cities in Europe as possible. Over the past years, the Sustainable Urban Mobility Plan (SUMP) concept has been worked into various European Union documents (e.g. Action Plan on Urban Mobility-COM, 2009, 490; White Paper Roadmap-COM, 2011, 0144), as well as in European projects, notably those financed by the IEE. The current and detailed information on SUMP and its applications are all available on www. mobilityplans.eu website. There too, among other things, one can find guides on how to create SUMP and information about the proposed methodology. SUMP can be partly combined with the previous mobility and transport plans, which have emerged in cities (with specific size and characteristics) in recent years in order to cope with transport problems. Therefore, the ENCLOSE along with the ongoing SULPiTER projects view the sustainable urban logistics plan as an essential part of the urban mobility outlook and aim to develop the SULP in every project city, while respecting the SULP compatibility with SUMP (ENCLOSE, 2015).

SULP in practice is a detailed plan of management of urban logistics processes, a medium-term-solutions design. It is also a tool to define a common vision, a tool for designing a set of appropriate measures, and ultimately a tool to reduce air and noise pollution and energy consumption.

The SULP methodology is defined following the SUMP approach. SULP elements are (Ambrosino, Liberato, Pettinelli, 2015):

1. Setting the target.

2. Urban mobility scenario and priorities.

3. Analysing context and logistic processes.

4. Setting the logistics baseline requirements and baselines.

5. Appropriate measures and services versus requirements.

6. Design of identified solutions.

7. Business model, actor role and responsibility.

8. Evaluation of services/solutions and impacts.

9. Obligations, implementation plan.

10. Promotion and communication plan.

11. Action plan for the adoption of SULP.

Every element of the above has to include justification, tasks, times, and methods.

The SULPiTER project, lasting from 2016 to 2019, was supposed to support policymakers in improving their understanding of FUAs commodity phenomena in the energy and environmental perspective. The project increased their capacity to plan urban mobility for freight transport in order to develop and adopt sustainable urban logistics plans. Policymakers in Bologna, Budapest, Poznan, Brescia, Stuttgart, Maribor, and Rijeka engaged in cooperation with other local, regional, and national non-partner authorities and with technical partners. 
The preparation of work under each SULP was based on (Foltyński, 2018):

1. Understanding

1.1. Benchmark analysis and research on trends and scenarios defined the scope at EU level of what each partner was going to do at a local level.

1.2. At a local level, the information on each FUA in terms of transport demand was faced by the developed tool.

2. Governance building

2.1. Stakeholders identification and engagement (both private and public sectors)

2.2. Freight Quality Partnership to co-create the plans

3. SULPs Development

3.1. Benchmark analysis and state of the art in FUAs

3.2. SULP development in each FUA

3.3. Enlarge transfer programme: to non-partners institutions (e.g. University of Gdańsk).

The partners worked to build the potential of transnational policy and to develop transnational analytical and management tools, thereby contributing to the improvement and adoption of policies for future sustainable energy and sustainability in Freight transport in Central European FUAs. In May 2019, the project ended, and all seven cities were preparing their SULP. Each of them was written on the basis of research in the area - traffic, suppliers, LSP, and business at the location. Depending on the conditions, profiled measures were introduced (SULPiTER, 2019).

The NOVELOG project in the years 2015-2018 served to enable cities to implement effective and sustainable policies and to facilitate cooperation between stakeholders in the field of sustainable urban logistics in order to provide knowledge and understanding of urban freight distribution and business travel.

The aim of the project was to strengthen the capacity of local authorities and stakeholders to create a sustainable policy by providing tools to manage the "implementation chain" (problem capture-decision-planning-testing- assessment - corrections - implementation). The overall objective of the project is a sustainable transport policy, with a better understanding of cost-effective strategies, measures, and business models to reduce the carbon footprint of logistics operations in cities. The NOVELOG project focused on providing knowledge and understanding of the distribution of goods and business travel by providing guidance on the implementation of effective and sustainable policies and measures. These guidelines support the selection of the most optimal and used solutions for urban transport and transport services and facilitate stakeholder cooperation and development, field testing, and transfer of best governance and business models.

The key concept of the project was to initiate and enable the formulation of urban logistics policy and decision-making in the framework of sustainable urban mobility planning, as well as to support the implementation of appropriate policies and measures. This was achieved through the guidance of policymakers based on sustainable business and logistic models and facilitating cooperation and consensus between stakeholders.

The triangular pyramid (Fig.1) presents interests and interactions between stakeholders. Municipal authorities (policymakers) are placed on top of the vortex 
as central stakeholders. Other stakeholders are divided into broadcasters/recipients, logistic service providers (freight forwarders, operators) and society (citizens).

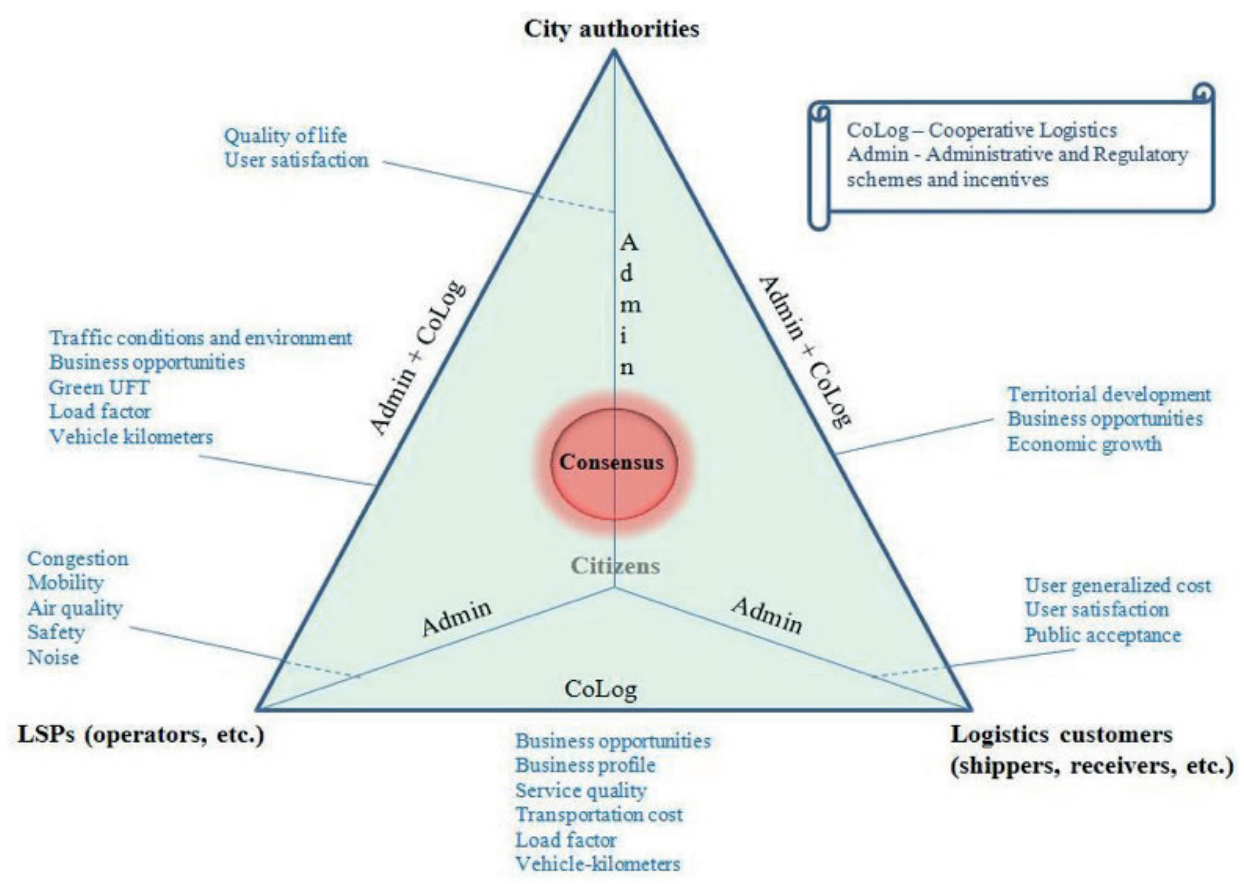

Figure 1. Stakeholders interactions pyramid Source: (NOVELOG, 2016)

The pyramid is a cooperation scheme for each pair of stakeholders, assuming "administrative and regulatory programmes and incentives" (admin) and/or "logistical cooperative" (CoLog), which consist of two concepts where all policies and the remedial measures discussed in the project are grouped together. The consensus is placed at the centre of the pyramid, as it constitutes the main ambition of creating the SULP, which is implemented in each work package by building a platform for exchanging information and knowledge, at different levels, concerning urban logistics.

The communication platform or the freight quality partnership can be used to achieve consensus. These tools allow for the accumulation of factors influencing urban freight transport (UFT) and the identification and collection of key performance indicators (KPIs) and methodologies used to assess the effectiveness of policies and measures for each stakeholder group. It shall disclose the objectives, priorities, and views of stakeholders and the future development plans of the UFT as well as initiate an open discussion arena that will evolve into a common decision-making process.

The results of selected or tested policies and measures shall be assessed on the basis of an integrated evaluation framework based on stakeholder objectives, priorities, and perceptions. The most suitable, enforceable method of evaluation 
is employed by each city, depending on the characteristics of the city as well as the elaborated and implemented multilateral decision-making techniques from multiple parties.

Based on pilot cases and case studies, a guide to best practices has been created that has increased knowledge of the conditions in a given area to better coordinate and manage these movements. The project investigated a number of systems and practices such as incentives for low-emission vehicles, overnight deliveries, parking law enforcement and service cooperation programmes, rescheduling of deliveries, consolidation in existing systems, etc.

The NOVELOG project developed a toolkit that helps cities to identify measures implemented in other similar cities and facilitate the selection of the most suitable measure or combination of measures for implementation. The toolkit helps the cities focus on the specific measures that would provide the greatest benefit to the city or to specific impact areas that are a city's priority.

As seen in Figure 2, the toolkit allows the user to filter the available measures in the database using a set of city parameters such as objectives, problems, city morphology, UFT logistics profile, UFT market, key stakeholders, and measures of implementation. A drop-down list for each of these parameters makes the experience as simple as possible for the user to select the most appropriate parameters and search for suitable measures implemented in other cities. Users may even filter results based on specific criteria to see impacts achieved elsewhere.

\section{novelog}

Typology Search Filters
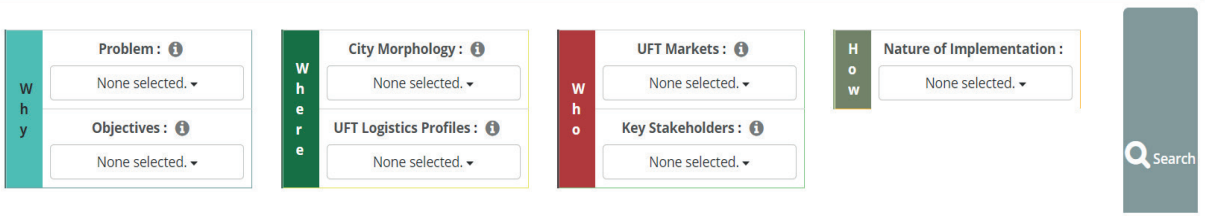

Figure 2. NOVELOG city logistics tool

Source: (NOVELOG, 2016)

To use this tool you need to select the suitable parameters (Fig. 3) and simply press "search" to see the list of measures and impacts implemented in cities with the same parameters.

The NOVELOG Toolkit also allows users to see the impacts of specific measures. The Toolkit also provides information on where and when this measure was implemented and what was its impact. The results for the chosen parameters (Gdańsk features) are shown in Figure 4. 


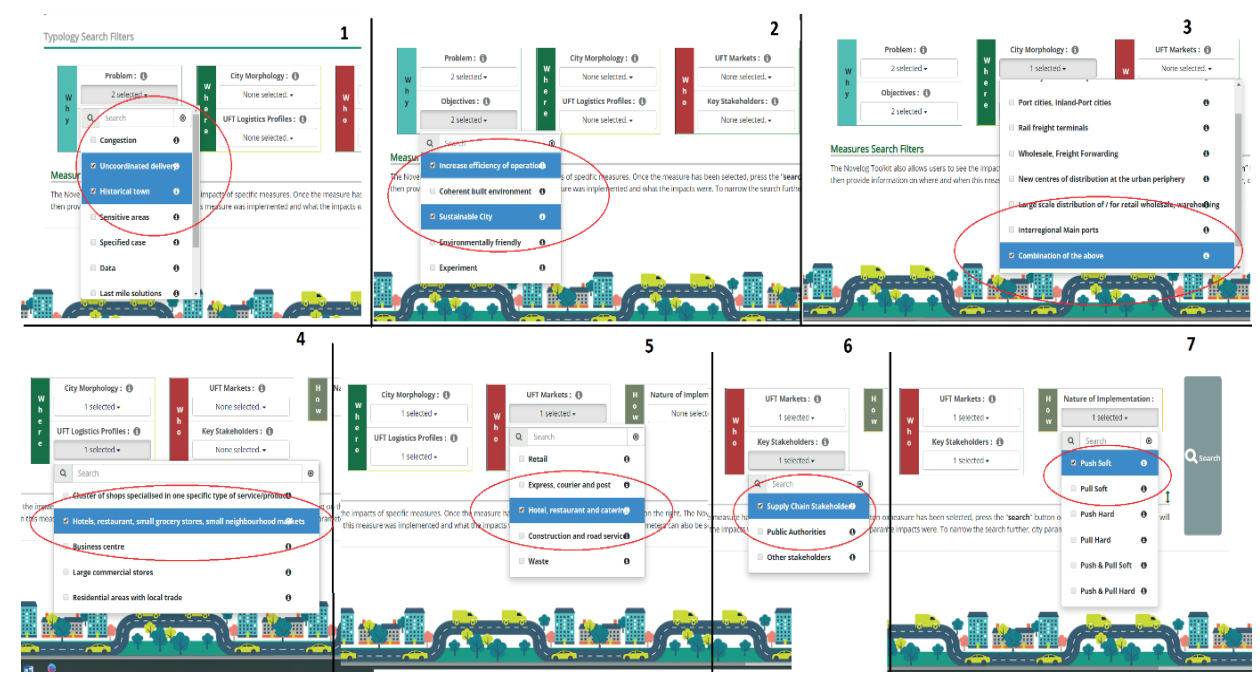

Figure 3. NOVELOG city logistics tool options (example) Source: (NOVELOG, 2016)

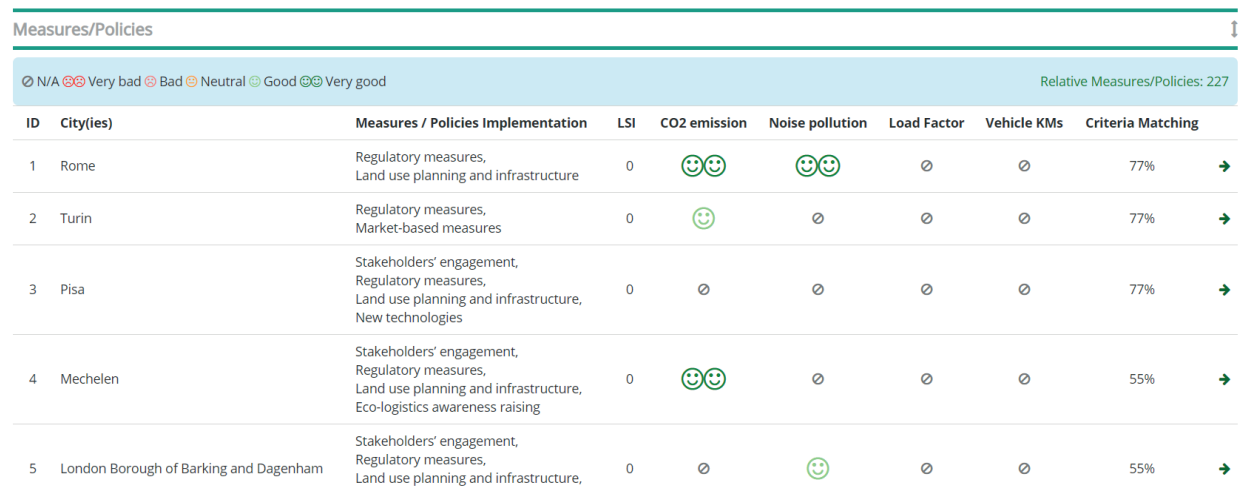

Figure 4. NOVELOG tool results (example)

Source: (NOVELOG, 2016)

\section{Conclusions}

For now, only a dozen cities have their own SULP, but thanks to the outcome of the projects NOVELOG, ENCLOSE and SULPiTER, and enlarged transfer program, good practices applied in projects can be spread and reproduced. Every city requires different measures; therefore, not every solution can be used anywhere. The decision on the selection of measures should be preceded by research and surveys together with stakeholders' engagement. However, following the roadmap adopted by SULPiTER, sustainable goals can be achieved with the satisfaction 
of all stakeholders. Without implementing a sustainable urban logistics plan, the ambitious goals of the White Paper on Transport cannot be achieved.

\section{References}

Ambrosino, G, Liberato, A., Pettinelli, 2015, I., Sustainable Urban Logistics Plans (SULP) Guidelines, Civitas Forum, Ljubljana.

ENCLOSE, 2015, ENCLOSE Project Website, http://www.enclose.eu, access: 30.04 .2019 Foltynski, M., 2018, New challenges for transport systems beyond 2020 -SULPiTER project, $3^{\text {rd }}$ International Conference Green Cities 2018 - Green Logistics for Greener Cities, 13-14 September 2018, Szczecin, Poland.

NOVELOG, 2016, NOVELOG Project Website, http://novelog.eu, access: 1.05.2019 SULPiTER, 2019, Sulpiter Project Website, https://www.interreg-central.eu/Content.Node/SULPiTER.html, access: 29.04.2019

\section{Corresponding author}

Maria Matusiewicz can be contacted at: m.matusiewicz@ug.edu.pl 